\title{
Las condiciones laborales de las y los colaboradores salvadoreños en el contexto del Covid-19
}

\author{
Marta Irene Flores Polanco a \\ José Adán Vaquenano Amaya ${ }^{b}$ \\ Marcos Antonio Morán Valencia c
}

Resumen - La presente comunicación aborda la concepción del trabajo decente a partir de las condiciones de oportunidad, psicológicas, de tiempo y productividad en que las y los colaboradores de las empresas salvadoreñas están desarrollando sus actividades laborales en el contexto de la pandemia COVID-19, así como sus temores y expectativas en cuanto al retorno al trabajo bajo la nueva normalidad de las personas que trabajan en las empresas formales públicas y privadas que desarrollan sus actividades en modalidad presencial y/o remota desde casa; las cuales han sido analizadas desde un enfoque de género y contrastándolo con el ideal del Trabajo Decente. Los resultados llevan a concluir que las mujeres están siendo sobrecargadas laboral, emocional y psicológicamente con lo cual se pone en riesgo su integridad física.

Palabras clave - Condiciones Laborales, Género, Expectativas, COVID-19, Trabajo Decente.

Abstract - This communication addresses the conception of decent work based on the conditions of opportunity, psychological, time and productivity in which the collaborators of Salvadoran companies are developing their work activities in the context of the COVID-19 pandemic, as well as their fears and expectations regarding the return to work under the new normal of people who work in formal public and private companies that carry out their activities in person and/or remotely from home; which have been analyzed from a gender perspective and contrasting it with the ideal of Decent Work. The results lead to the conclusion that women are being overloaded at work, emotionally and psychologically, thereby putting their physical integrity at risk.

Keywords - Working Conditions, Perception, Gender, COVID-19, Decent Work.

\section{CÓMO CITAR \\ HOW TO CITE:}

Flores-Polanco, M. I., Vaquerano-Amaya, J. A. y Morán-Valencia, M. A. (202I).

Las condiciones laborales de las y los colaboradores salvadoreños en el contexto del Covid-19. Interconectando Saberes, (12), 10I-II5. https://doi.org/10.25009/is.v0il2 .2708

Recibido: 31 de mayo de 2021 Aceptado: 5 de julio de 2021 Publicado: 20 de julio de 2021

a Universidad Centroamericana José Simeón Cañas, El Salvador. E-mail: miflores@uca.edu.sv

(iD) 0000-0002-2285-6104

b Universidad Centroamericana José Simeón Cañas, El Salvador. E-mail: avaquerano@uca.edu.sv

c Universidad Centroamericana José Simeón Cañas, El Salvador. E-mail: mamoran@uca.edu.sv 


\section{INTRODUCCIÓN}

La crisis sanitaria y económica provocada por la pandemia Covid-19, además de haber evidenciado la fragilidad de los sistemas de salud y económico a nivel mundial, ha profundizado aún más las ya amplias y reconocidas desigualdades en el ámbito económico, social y laboral alrededor del mundo.

En efecto, el Covid-19 ha impactado en las desigualdades de género agudizando la inequidad hacia la mujer (Guterres, A., 2020), pues durante la crisis del coronavirus se agudiza el trabajo de cuidados no remunerados y la doble $\circ$ triple jornada laboral (Mujeres, O.N.U., 2020 a) pues alguien tiene que hacerse cargo de las labores domésticas del hogar, de preparar los alimentos, del cuido de los hijos y el apoyo con las tareas escolares, por mencionar algunas.

Todo lo anterior conduce a una situación de fatiga emocional y física por la carga extra de trabajo, sin mencionar el hecho del notable aumento de la violencia doméstica, que viene a aumentar más la precariedad de la situación que ellas viven actualmente.

Estamos ante una situación de una evidente violencia psicológica y emocional en contra de las mujeres, dado que están siendo sometidas a situaciones o conductas que directa $\circ$ indirectamente les están ocasionando algún tipo de daño emocional o les disminuye su autoestima, con lo cual se está perjudicando su pleno desarrollo.

Al evaluar dicha situación desde la perspectiva del trabajo decente, se evidencia una violación al mismo, puesto que no se está desarrollando en condiciones de libertad (Tadjoeddin, 2019), equidad, seguridad (Gálvez, E., Gutiérrez, E., Picazzo, E. y Osorio, J., 2016), respeto a la dignidad humana (Benedicto XVI, 2009) y libre de discriminación (Cornelio-Landero, 2019) y que ponga al centro a la persona humana (Pravin, 2019).

\section{MARCo CONTEXTUAL}

Al estudiar los datos demográficos de El Salvador, se evidencia la amplia segregación de género que existe, misma que es común en el resto de los países latinoamericanos.

La Encuesta de Hogares de Propósitos Múltiples (EHPM) para el 2019 señala que las mujeres representan el $53 \%$ del total de la población salvadoreña; sin embargo, cuando se mide la tasa global de participación aproximadamente 5 de cada 10 mujeres están ocupadas u ofertando su fuerza de trabajo, ante un notable $80.5 \%$ que representan los hombres, con lo cual sus oportunidades de desarrollo comienzan a reducirse, a pesar que las mujeres presentan una escolaridad promedio un poco arriba que los hombres ( 8.8 años en mujeres y 8.4 años en hombres).

Al estudiar la población ocupada (personas que tienen un trabajo del cual obtienen una remuneración 0 ganancia) el $58.6 \%$ son hombres y el $4 \mathrm{I} .4 \%$ son mujeres. Esta población reporta una brecha salarial entre hombres y mujeres ya que al tomar como base el salario promedio mensual, las mujeres reciben $\$ 67.29$ (18\%) menos en relación con salario que recibe el hombre, dado que ellas en promedio ganaban $\$ 306.1$ I en comparación a $\$ 373.40$ recibidos por ellos (DIGESTYC, 2019). Por lo tanto, se reafirma la brecha salarial existen entre hombres y mujeres, que es reflejo del mercado laboral mundial y que persiste en todo tipo de organizaciones (Reyes Sánchez et al., 2018).

Las ramas de actividades económicas en que trabajan la mayor cantidad de mujeres continúan siendo las relacionadas a las actividades de cuido y atención 
como comercio, hoteles y restaurantes (44.4\%), industria manufacturera (16.7\%) y el servicio doméstico (II.7\%) (DIGESTYC, 20I9).

Cabe indicar que El Salvador ha avanzado en el camino de aumentar la participación de la mujer en el mercado laboral, mediante programas como Ciudad Mujer, donde se han diseñado e implementado modelos de atención y apoyo integral a las mujeres (PNUD, 20I5).

Mediante este y otros programas se propician entornos socioeconómicos activos donde hombres y mujeres puedan desarrollar actividades productivas y comerciales, ya sea de forma individual o colectiva, que les permitan mejorar sus condiciones de vida, mediante la obtención de una retribución justa y equitativa por el esfuerzo realizado (Organización Internacional del Trabajo, 2004).

En el contexto actual de la pandemia, las brechas existentes en los países y entre los países se acentuaron y El Salvador no fue la excepción, ya que cuando se estableció el estado de emergencia nacional bajo el decreto $N^{\circ} 593$ donde se limitaba la circulación a nivel nacional, las y los colaboradores de las empresas que no se encontraban dentro de los sectores que no se encontraban desempeñando funciones en primera línea para combatir la pandemia como salud, seguridad y obras públicas, se vieron obligadas a guardar cuarentena en sus casa y las y los empresarios buscaron mecanismos para salvaguardar sus operaciones implementando la modalidad del trabajo desde casa (para las actividades que aplicaban) y el trabajo presencial con una disminución cuantiosa de las y los colaboradores presentes en las empresas. Dicha situación se extendió hasta junio 2020 cuando mediante el decreto 31 se iniciaron las fases de reactivación económica.
Durante el estado de emergencia que llevó a la cuarentena domiciliar obligatoria muchas empresas despidieron a sus colaboradores, suspendieron los contratos de trabajo o enviaron a sus colaboradores a realizar el trabajo desde casa con una reducción de salario y/o prestaciones, en este escenario fueron las mujeres, los jóvenes y los adultos mayores de 43 años los que sufrieron más este impacto (Vaquerano-Amaya et al., 2020).

\section{MARCO TEÓRICO}

Desde su concepción, el trabajo decente contempla como temas transversales el desarrollo, la equidad de género y la dignidad en el trabajo (Somavía, 1999), para todas y todos los trabajadores tanto del sector formal como informal.

Pues toda la población en edad de trabajar debe tener garantizadas las posibilidades de encontrar un trabajo y formar parte del mercado laboral en igualdad de condiciones, esto implica tener un ingreso justo que garantice la subsistencia de las y los colaboradores y sus familias. Lo anterior no debería de menoscabar la generación de espacios adecuados para que la persona trabajadora pueda conciliar sus responsabilidades familiares con las responsabilidades del trabajo; pues toda jornada laboral, sin importar su naturaleza, debe tener un parámetro de horas adecuadas para la realización de las labores y cuidar que no afecte la salud física y mental de las y los colaboradores, ni afectar el tiempo que le dedican a su vida familiar y social (Anker et al., 2003).

En la mayoría de los países las responsabilidades del cuidado del hogar, niñez y personas adultas las realizan las mujeres, estos roles de género que la sociedad les ha atribuido a lo largo de la historia, se han 
convertido en un desafío pues, aunque su participación en el mercado laboral es elevada, los roles generan discriminación e inequidad hacia ellas.

Otro desafío para las organizaciones lo representa el velar por generar condiciones de equidad y trato igualitario, deben mantener un clima laboral motivante, participativo e inclusivo para que sus colaboradores sean productivos, se encuentren satisfechos e impacten en la productividad y eficiencia de la organización (Pozo-Pérez, 2015).

Entre las condiciones por las que debe velar la organización, se encuentran las de oportunidad las cuales se orientan a estudiar si las y los colaboradores logran un equilibrio entre su vida laboral, familiar y social, para ello se observan los diferentes roles y responsabilidades que pueden desempeñar, adicional al trabajo remunerado, así como el trato equitativo de las empresas hacia quienes realizan esta multiplicidad de roles.

Además, el análisis de las condiciones psicológicas, especialmente en estos tiempos de pandemia cobran mayor relevancia, pues miden la atención o apoyo psicológico que las organizaciones brindan para afrontar los problemas de estrés, así como la atención a las diferentes preocupaciones y necesidades que tengan las y los colaboradores, también se estudia la sobrecarga de tareas que poseen y la presencia de los síntomas de estrés que pueden experimentar.

También es importante el estudio de las condiciones de tiempo y productividad para evaluar el respeto al cumplimiento de los horarios de trabajo y las pausas para alimentación y así evitar la sobrecarga de tareas, el estrés y lograr el tan anhelado equilibrio entre la vida laboral-familiar-social sin disminuir la productividad de las y los colaboradores.

Por lo tanto, procurar que el mercado laboral provea más y mejores oportunidades de empleo para mujeres y jóvenes, con ingresos adecuados y justos, donde se cumplan y respeten las leyes laborales y no exista sobrecarga de trabajo, sino más bien se vele por un equilibrio entre el trabajo y la vida familiar, es un deber de los estados y un derecho de las personas que integran la población en edad de trabajar.

\section{MARCO METODOLÓGICO}

Tomando como referencia lo antes expuesto, se vuelve vital investigar y profundizar en las condiciones en que las y los colaboradores de las empresas salvadoreñas desarrollan sus actividades laborales en el contexto de la pandemia COVID-19, así como los posibles temores $y$ las expectativas que poseen en cuanto al retorno al trabajo bajo la nueva normalidad; tanto para las personas que trabajan en las empresas formales públicas y privadas que desarrollan sus actividades en modalidad presencial y/o remota desde casa.

Por lo que durante el período de mayo a septiembre de 2020 se realizó la investigación: Una aproximación al clima organizacional en las empresas de El Salvador bajo el contexto de la pandemia COVID-19 (Vaquerano-Amaya et al., 2020), que abarcó todo el territorio de la república de El Salvador y se obtuvo la respuesta de una muestra de 518 colaboradores contratados de manera formal por parte de empresas del sector privado y público. En dicha investigación se estudiaron 7 condiciones del clima organizacional de las cuales, para este artículo, se retoma la base de datos de 
3 condiciones, las expectativas y los temores, presentando nuevos hallazgos.

En tal sentido, el objetivo del presente artículo es presentar las condiciones de oportunidad, psicológicas, de tiempo y productividad de las y los colaboradores salvadoreños en el contexto del Covid19, así como de las expectativas para el retorno al trabajo y las preocupaciones o temores en el contexto de la nueva normalidad, abordando su estudio desde una perspectiva de género y contrastándolo con el ideal del Trabajo Decente.

\section{PRESENTACIÓN DE RESULTADOS}

\section{Las condiciones de oportunidad de las y los colaboradores en modalidad de trabajo presencial y remoto desde casa}

Los resultados de las condiciones de oportunidad para la modalidad de trabajo presencial se presentan en la Tabla I, y han sido analizadas en base a las respuestas que han proporcionado las y los colaboradores de las empresas públicas y privadas a partir de las dos preguntas siguientes: ¿Ha logrado un equilibrio entre su vida laboral y su vida familiar? y ¿Ha logrado un equilibrio entre su vida laboral y su vida social?

\section{Tabla I}

Equilibrio entre la vida laboral, familiar y social en modalidad de trabajo presencial

\begin{tabular}{|c|c|c|c|c|c|c|}
\hline \multirow{7}{*}{ 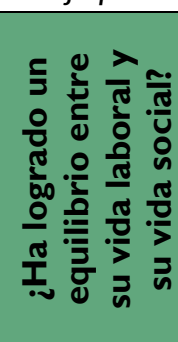 } & \multicolumn{6}{|c|}{$\begin{array}{l}\text { ¿Ha logrado un equilibrio entre su vida } \\
\text { laboral y su vida familiar? }\end{array}$} \\
\hline & & & \multicolumn{2}{|c|}{ Hombre } & \multicolumn{2}{|c|}{ Mujer } \\
\hline & & & $\mathrm{Si}$ & No & $\mathrm{Si}$ & No \\
\hline & \multirow{2}{*}{ Hombre } & $\mathrm{Si}$ & $39(78 \%)$ & 5 & & \\
\hline & & No & 11 & 10 & & \\
\hline & \multirow{2}{*}{ Mujer } & $\mathrm{Si}$ & & & $42(85 \%)$ & 8 \\
\hline & & No & & & 7 & 19 \\
\hline
\end{tabular}

logrado alcanzar un equilibrio entre su vida laboral, familiar y social en comparación con los hombres. Situación que se puede deber al hecho que a las mujeres por su multiplicidad de roles se ven obligadas a manejar una gran cantidad de responsabilidad por lo que hacen uso mayoritariamente de llamadas o a través del uso de las redes sociales a diferencia de los hombres, de quienes se puede afirmar que por un lado tienen menos roles en el hogar y por otro lado son más dados a las relaciones cercanas. En tal sentido, de acuerdo con los datos presentados y las razones antes expuestas, la pandemia Covid-19 ha impactado en los dos factores analizados más a los colaboradores que a las colaboradoras.

En lo que respecta a los resultados de las condiciones de oportunidad para la modalidad de trabajo remoto desde casa, se han analizado las respuestas que han proporcionado las y los colaboradores ante los cuestionamientos siguientes: $\mathrm{iHa}$ logrado un equilibrio entre su vida laboral y su vida familiar?, ¿Ha logrado un equilibrio entre su vida laboral y su vida social?, ¿Tiene responsabilidad del cuidado de los hijos?, ¿Tiene responsabilidad de realizar las labores domésticas? y ¿Tiene responsabilidad de la preparación de los alimentos?

Los datos reflejados en la Tabla 2 vuelven a poner de manifiesto, igual que para el caso de las y los colaboradores que están haciendo trabajo presencial, que son las mujeres las que en su mayoría han logrado alcanzar un equilibrio entre su vida laboral, familiar $y$ social (89\%) en comparación con los hombres (85\%).

De acuerdo con los datos que se presentan en la Tabla I, son las mujeres las que en su mayoría han 
Tabla 2

Equilibrio entre la vida laboral, familiar y social en modalidad remota

\begin{tabular}{|c|c|c|c|c|c|c|}
\hline \multirow{7}{*}{ 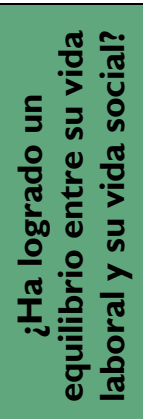 } & \multicolumn{6}{|c|}{$\begin{array}{l}\text { ¿Ha logrado un equilibrio entre su vida } \\
\text { laboral y su vida familiar? }\end{array}$} \\
\hline & & & \multicolumn{2}{|c|}{ Hombre } & \multicolumn{2}{|c|}{ Mujer } \\
\hline & & & $\mathrm{Si}$ & No & $\mathrm{Si}$ & No \\
\hline & \multirow{2}{*}{ Hombre } & $\mathrm{Si}$ & 81 (85\%) & 7 & & \\
\hline & & No & 14 & 30 & & \\
\hline & \multirow{2}{*}{ Mujer } & $\mathrm{Si}$ & & & 92 (89\%) & 9 \\
\hline & & No & & & 11 & 57 \\
\hline
\end{tabular}

\section{Tabla 3}

Responsabilidad del cuido de los hijos y de ayudar con sus tareas escolares

\begin{tabular}{|c|c|c|c|c|c|c|}
\hline \multirow{7}{*}{ 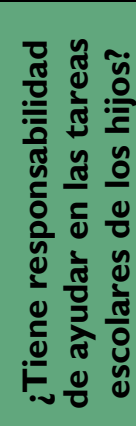 } & \multicolumn{6}{|c|}{$\begin{array}{l}\text { ¿Tiene responsabilidad del cuidado de } \\
\text { los hijos? }\end{array}$} \\
\hline & & & \multicolumn{2}{|c|}{ Hombre } & \multicolumn{2}{|c|}{ Mujer } \\
\hline & & & $\mathrm{Si}$ & No & $\mathrm{Si}$ & No \\
\hline & \multirow{2}{*}{ Hombre } & $\mathrm{Si}$ & $26(65 \%)$ & 6 & & \\
\hline & & No & 14 & 74 & & \\
\hline & \multirow{2}{*}{ Mujer } & $\mathrm{Si}$ & & & 4 ( $(72 \%)$ & 0 \\
\hline & & No & & & 16 & 99 \\
\hline
\end{tabular}

Los datos presentados en la Tabla 3 indican que del $100 \%$ de las mujeres que tienen la responsabilidad del cuido de los hijos, a un $72 \%$ de ellas también les recae la responsabilidad de ayudarles con las tareas escolares, en relación con un $65 \%$ de los hombres que también dicen cumplir con ambas responsabilidades. Al respecto, es importante indicar que son las mujeres colaboradoras de las empresas salvadoreñas las que en su mayoría deben de cumplir con el triple rol de ser empleadas, cuidadoras de sus hijos y el ayudar en sus tareas escolares; situación que, sin lugar a duda, está creándoles una sobrecarga de trabajo, que a la larga pude afectar su salud física, emocional y psicológica.

\section{Tabla 4}

Responsabilidad de realizar las labores domésticas y de preparar los alimentos

\begin{tabular}{|c|c|c|c|c|c|c|}
\hline \multirow{7}{*}{ 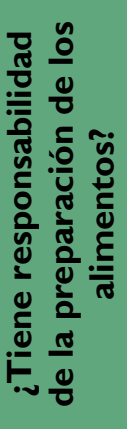 } & \multicolumn{6}{|c|}{$\begin{array}{l}\text { ¿Tiene responsabilidad de realizar las } \\
\text { labores domésticas? }\end{array}$} \\
\hline & & & \multicolumn{2}{|c|}{ Hombre } & \multicolumn{2}{|l|}{ Mujer } \\
\hline & & & $\mathrm{Si}$ & No & $\mathrm{Si}$ & No \\
\hline & \multirow{2}{*}{ Hombre } & $\mathrm{Si}$ & $74(65 \%)$ & 2 & & \\
\hline & & No & 39 & 19 & & \\
\hline & \multirow{2}{*}{ Mujer } & $\mathrm{Si}$ & & & $123(87 \%)$ & 3 \\
\hline & & No & & & 18 & 29 \\
\hline
\end{tabular}

De nueva cuenta, los datos de la Tabla 4 evidencian el hecho que una vez más la crisis sanitaria y económica provocada por la pandemia Covid-19 está afectando mayoritariamente a las mujeres, dado que el $87 \%$ de las colaboradoras que hacen trabajo remoto desde casa manifiestan tener la responsabilidad de realizar las labores domésticas y la preparación de los alimentos, en comparación del $65 \%$ de hombres que dicen también cumplir ambos roles. Estos resultados, aunados a los anteriores, vuelven a poner de manifiesto la situación de sobrecarga de trabajo por la que están pasando las mujeres trabajadoras salvadoreñas.

Los resultados antes señalados presentan una situación lamentable, en lo que concierne a los factores de las condiciones de oportunidad analizadas, tanto para las y los colaboradores que están haciendo trabajo presencial como para las y los que están haciéndolo de manera remota desde casa, en el sentido que en sus organizaciones no se les está dando ningún tipo de trato especial, dada la multiplicidad de roles que deben jugar en el hogar, tal como se presenta en la Tabla 5, donde se refleja la percepción de las mujeres y hombres que están haciendo trabajo remoto desde casa. 
Tabla 5

Trato especial por considerar su multiplicidad de roles en el hogar

\begin{tabular}{|c|c|c|c|}
\hline \multicolumn{4}{|c|}{$\begin{array}{c}\text { Le dan algún trato especial por considerar su } \\
\text { multiplicidad de roles en el hogar? }\end{array}$} \\
\hline & $\mathrm{Si}$ & No & Total \\
\hline Hombre & 34 & $97(74 \%)$ & I3I \\
\hline Mujer & 39 & $119(75 \%)$ & 158 \\
\hline
\end{tabular}

Se trata sin lugar a duda de una situación que deja en evidencia el hecho de que las empresas están siendo poco solidarias para con sus colaboradoras y colaboradores, especialmente con ellas, pues son quienes están llevando una sobrecarga laboral y familiar que puede poner en riesgo su integridad física, emocional y psicológica y, por lo tanto, su desempeño laboral. Razón por la cual, sería recomendable que los líderes y tomadores de decisiones en las empresas públicas y privadas salvadoreñas analicen dicha situación, dado que, a la larga los problemas físicos, emocionales y psicológicos que afecten a sus colaboradores, terminarán también afectando el desempeño, productividad y competitividad de sus organizaciones.

\section{Las condiciones de oportunidad de las y los colaboradores en modalidad de trabajo presencial y remoto desde casa}

El aparecimiento del SARS-CoV-2, el confinamiento y la denominada "nueva normalidad" han impactado en la salud mental de mujeres y hombres. Sus efectos psicológicos se prevén que generará una segunda pandemia en el mundo.

Específicamente el estrés y la ansiedad han sido más marcadas en las mujeres, que como se mencionó anteriormente reportan mayor sobrecarga de tareas, responsabilidad en el cuidado de los hijos y las tareas domésticas.

Los datos que a continuación se presentan, pertenecen a las y los colaboradores que reportaban haber experimentado consecuencias psicológicas de entre las $15 \mathrm{I}$ personas que trabajan presencialmente $y$ 310 personas desde casa.

La Figura I evidencia que las mujeres que realizan trabajo presencial reportan mayores consecuencias psicológicas en cuatro factores de esta condición: estrés, ansiedad, dificultad de concentración y nerviosismo. Si bien es cierto que los hombres también reportan la misma situación, es importante evidenciar que sus porcentajes son minoritarios, con respecto al de las mujeres que duplican en la mayoría de los casos su prevalencia.

\section{Figura I}

Prevalencia de las condiciones psicológicas por sexo de las personas que realizan trabajo presencial

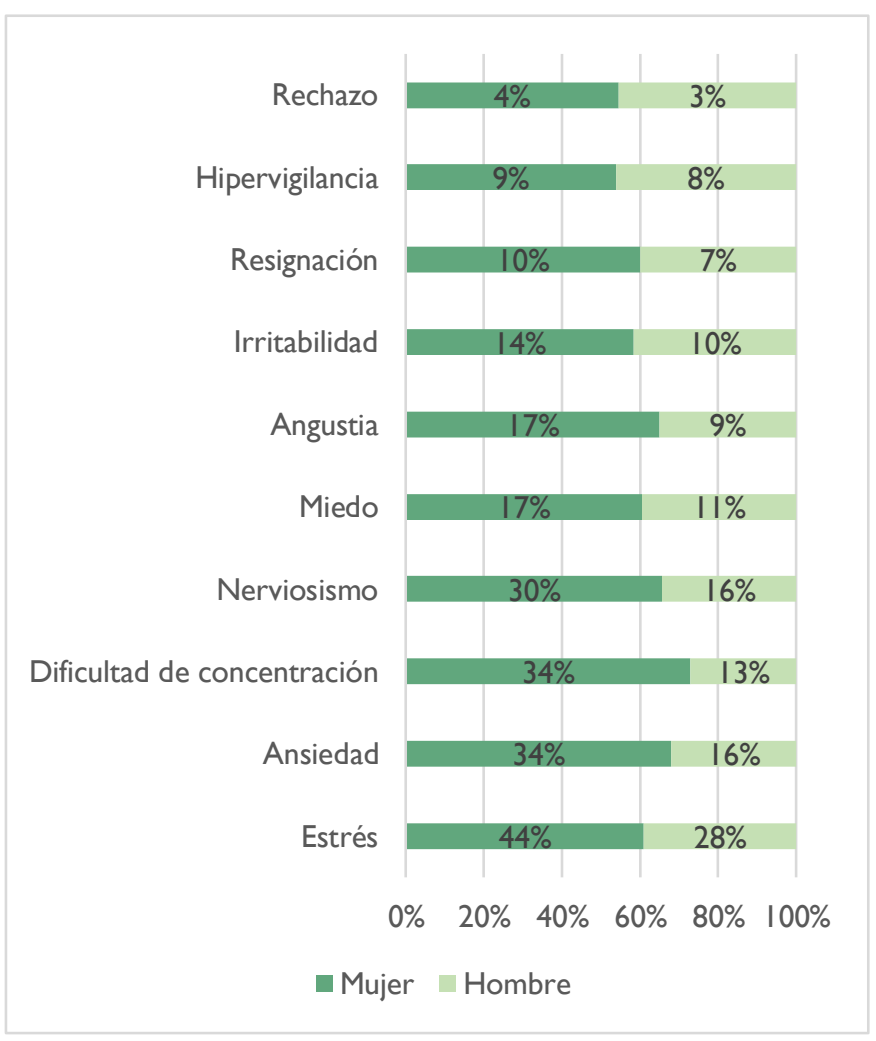

De acuerdo con la Figura 2, las mujeres poseen una prevalencia del estrés del $46 \%$ y $44 \%$ en las diferentes modalidades de trabajo, contrastado con un $29 \%$ y $28 \%$ de los hombres; pero llama la atención que 
las mujeres que trabajan desde casa han experimentado en mayor medida las consecuencias psicológicas con respecto a las mujeres que trabajan presencialmente en sus lugares de trabajo. Las mujeres que trabajan desde casa experimentan estrés, ansiedad, miedo, angustia e irritabilidad en porcentajes mayores que las mujeres que trabajan presencialmente; únicamente la dificultad de concentración y el nerviosismo presentan mayores porcentajes en las mujeres que trabajan presencialmente con respecto a las que trabajan desde casa. La irritabilidad tiene una diferencia porcentual del $14 \%$ en las mujeres que trabajan desde casa con respecto a las que realizan trabajo presencial. Las primeras además de sus labores tienen la responsabilidad de las actividades de cuido como el cuidado de los hijos, ayuda en las labores escolares, realización de las labores domésticas y la preparación de los alimentos, ejemplos de los roles atribuidos a las mujeres (estereotipos de género). La prevalencia mayor de la irritabilidad puede ser producto de esta multiplicidad de tareas que ejecutan.

Por otro lado, de un total de 169 mujeres que realizan trabajo desde casa, el $60 \%$ reportan que han implementado pausas para descansar del trabajo que realizan, pero un $40 \%$ no lo ha hecho (20 puntos por debajo) y, de un total de 173 mujeres que respondieron que sí les han brindado capacitaciones o información para el cuidado de su salud mental, un $49 \%$ manifestaba que no.

Volviendo a la Figura 2, las mujeres que realizan trabajo presencial reportan una diferencia porcentual mayor en el nerviosismo (13\%) y dificultad de concentración (6\%) con respecto a las mujeres que realizan su trabajo desde casa, esto podría tener sus causas debido a la constante preocupación por su multiplicidad de roles que desempeñan en el hogar y que tienen que asumir a su vuelta a casa, así como el temor del posible contagio de la enfermedad al resto de miembros del hogar. Adicionalmente de 57 mujeres que trabajan presencialmente un $44 \%$ reporta tener sobrecarga de tareas en el trabajo.

\section{Figura 2}

Prevalencia de condiciones psicológicas en mujeres y hombres

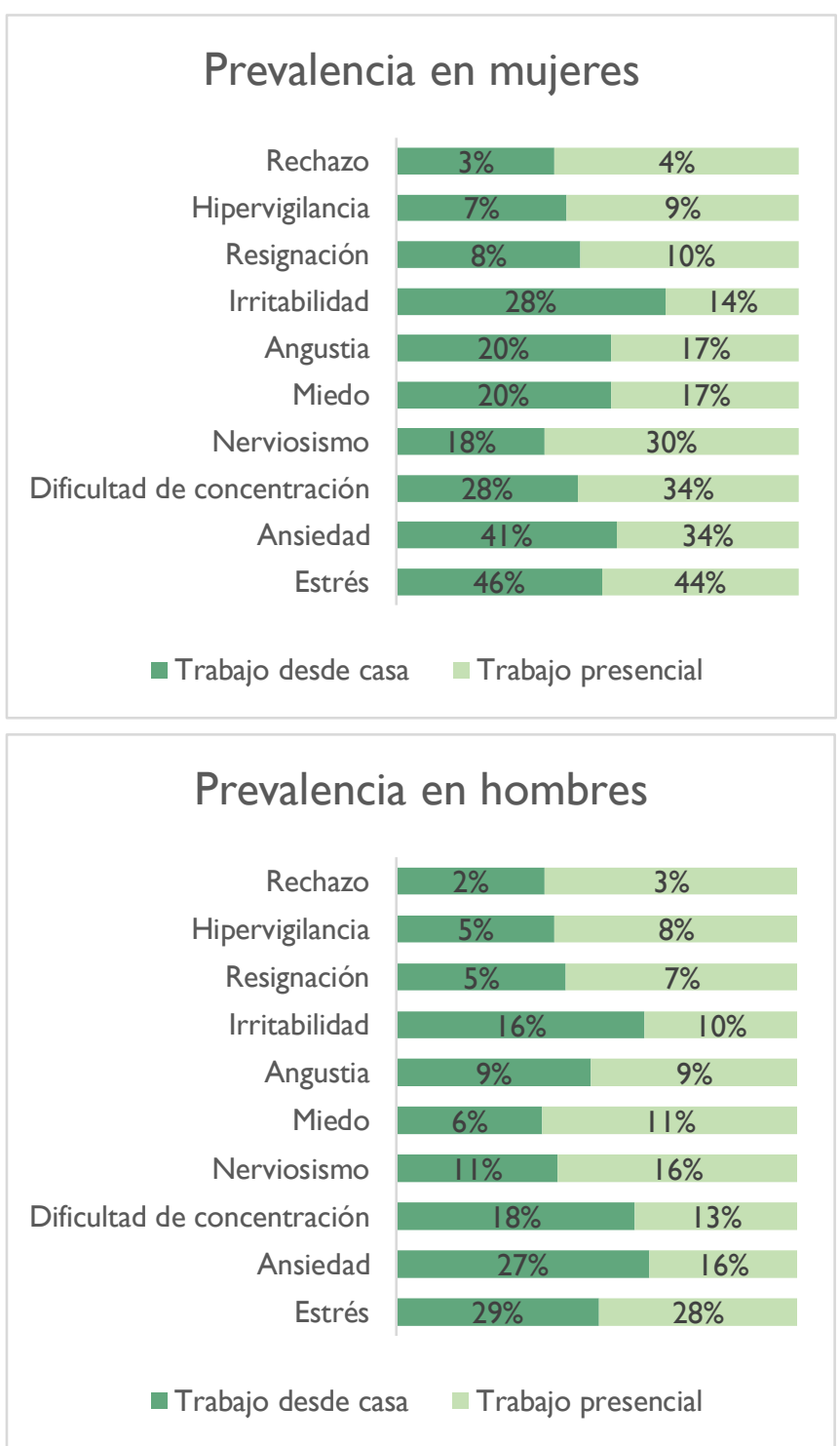

Por otra parte, 78 mujeres respondieron a la pregunta de si se han generado espacios dentro de la organización para reportar sus necesidades y preocupaciones $y$, un $53 \%$ de ellas reportan que no. 


\section{Tabla 6}

Condición de melancolía y/o depresión en modalidad de trabajo presencial

\begin{tabular}{|c|c|c|c|c|c|c|}
\hline \multirow{7}{*}{ 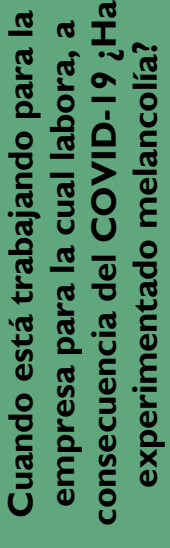 } & \multicolumn{6}{|c|}{$\begin{array}{l}\text { Cuando está trabajando para la } \\
\text { empresa para la cual labora, a } \\
\text { consecuencia del COVID-19 ¿Ha } \\
\text { experimentado depresión? }\end{array}$} \\
\hline & & & & ombre & & Mujer \\
\hline & & & $\mathrm{Si}$ & No & $\mathrm{Si}$ & No \\
\hline & Hombre & $\mathrm{Si}$ & 4 & $3(5 \%)$ & & \\
\hline & 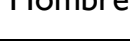 & No & 9 & 54 & & \\
\hline & Muin & $\mathrm{Si}$ & & & 4 & $5(7 \%)$ \\
\hline & Trujer & No & & & 4 & 68 \\
\hline
\end{tabular}

Al respecto, en la Tabla 6 se presentan los resultados de la consulta de si habían experimentado condiciones de melancolía y depresión cuando están trabajando para la empresa para la cual laboran, en lo cual del 100\% de mujeres que no manifiestan sentir depresión, un 7\% de ellas muestra que sí percibe melancolía, en relación con un $5 \%$ que percibe el hombre.

\section{Tabla 7}

Condición de melancolía y/o depresión en modalidad de trabajo remoto desde casa

\begin{tabular}{|c|c|c|c|c|c|c|}
\hline 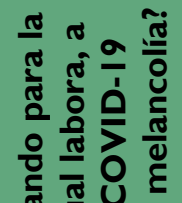 & \multicolumn{6}{|c|}{$\begin{array}{l}\text { Cuando está trabajando para la } \\
\text { empresa para la cual labora, a } \\
\text { consecuencia del COVID-19 ¿Ha } \\
\text { experimentado depresión? }\end{array}$} \\
\hline 늘 & & & \multicolumn{2}{|c|}{ Hombre } & \multicolumn{2}{|c|}{ Mujer } \\
\hline \multirow{5}{*}{ 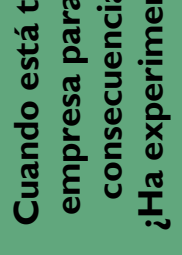 } & & & $\mathrm{Si}$ & No & $\mathrm{Si}$ & No \\
\hline & \multirow[t]{2}{*}{ Hombre } & $\mathrm{Si}$ & $\begin{array}{c}5 \\
(28 \%)\end{array}$ & 18 & & \\
\hline & & No & 13 & 100 & & \\
\hline & \multirow{2}{*}{ Mujer } & $\mathrm{Si}$ & & & $17(46 \%)$ & 23 \\
\hline & & No & & & 20 & 114 \\
\hline
\end{tabular}

Escenario que es totalmente diferente si se analiza con las personas que han realizado su trabajo desde casa (Tabla 7), donde del 100\% de mujeres que experimentan solo el factor de depresión al menos $46 \%$ dice también experimentar melancolía, número significativo si se compara con los resultados de los hombres ( $28 \%$ de ellos presentan ambas condiciones).

En esta situación pandémica, es bastante difícil, solo con el simple hecho de estar trabajando desde casa, tener las responsabilidades del hogar y que el colaborador y colaboradora tenga temores relacionados con la pérdida del puesto de trabajo y/o el cierre de las operaciones de las empresas; también están los que han experimentado la pérdida de compañeros de trabajo o familiares durante este tiempo de pandemia, causa sin lugar a duda de este tipo de emociones.

Es en este sentido, que se les consulto a los colaboradores y colaboradoras que realizan su trabajo en modalidad presencial, si en sus empresas existen grupos de autoayuda para aminorar el impacto psicológico, así como si existen canales para reportar el estrés crónico que pueden estar viviendo. Los resultados que se observan en la Tabla 8 indican que del $100 \%$ de los hombres y de las mujeres que no cuentan con un canal para reportar estrés, un $91 \%$ indica que tampoco cuentan con grupos de autoayuda, caso similar en las mujeres que en un $89 \%$ indican no contar con ninguna de las dos opciones para poder manejar o poder tener un soporte que le ayude a mejorar su salud psicológica.

Es alarmante que las empresas no estén prestando atención a situaciones de gran importancia como lo es la salud emocional y psicológica de sus colaboradores, dado que del $100 \%$ de hombres y mujeres que expresan que en sus organizaciones sí cuentan con canales para reportar estrés crónico solo el $63 \%$ de ellos manifiestan tener acceso a grupos de autoayuda para disminuir el impacto psicológico que el 
Covid-19 y sus efectos económicos y sociales que les están causando y así no verse afectados en la productividad de sus puestos de trabajo.

\section{Tabla 8}

Canales para reportar estrés crónico y existencia de grupos de autoayuda

\begin{tabular}{|c|c|c|c|c|c|c|}
\hline \multirow{7}{*}{ 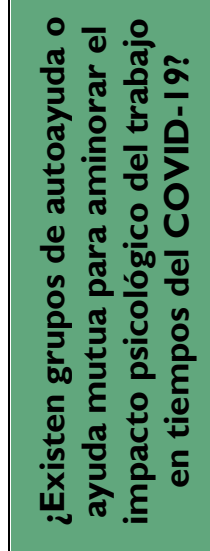 } & \multicolumn{6}{|c|}{$\begin{array}{l}\text { ¿Se han abierto canales para reportar } \\
\text { el estrés crónico que pueda estar } \\
\text { viviendo usted o sus compañeros de } \\
\text { trabajo? }\end{array}$} \\
\hline & & & \multicolumn{2}{|c|}{ Hombre } & \multicolumn{2}{|c|}{ Mujer } \\
\hline & & & $\mathrm{Si}$ & No & $\mathrm{Si}$ & No \\
\hline & \multirow{2}{*}{ Hombre } & $\mathrm{Si}$ & $\begin{array}{c}12 \\
(63 \%)\end{array}$ & 4 & & \\
\hline & & No & 7 & $\begin{array}{c}41 \\
(91 \%)\end{array}$ & & \\
\hline & \multirow[b]{2}{*}{ Mujer } & $\mathrm{Si}$ & & & $12(63 \%)$ & 6 \\
\hline & & No & & & 7 & $\begin{array}{c}50 \\
(89 \%)\end{array}$ \\
\hline
\end{tabular}

Los datos obtenidos de los factores de las condiciones psicológicas analizadas llevan a concluir que los líderes y tomadores de decisiones de las organizaciones deben darle la importancia debida a la gestión de la salud emocional y psicológica de las y los colaboradores, dado que si no se atienden debidamente pueden ocasionar un impacto negativo no solo en la salud física, por los posibles escenarios

de estrés crónico que pasan las y los colaboradores, sino por la afectación negativa en los resultados de la organización, ya que la satisfacción del personal puede experimentar una disminución significativa y esto puede llevar a una baja en la productividad (ver Figura 3).

\section{Figura 3}

Percepción por sexo de la gestión emocional y psicológica

\section{Percepción de los hombres}

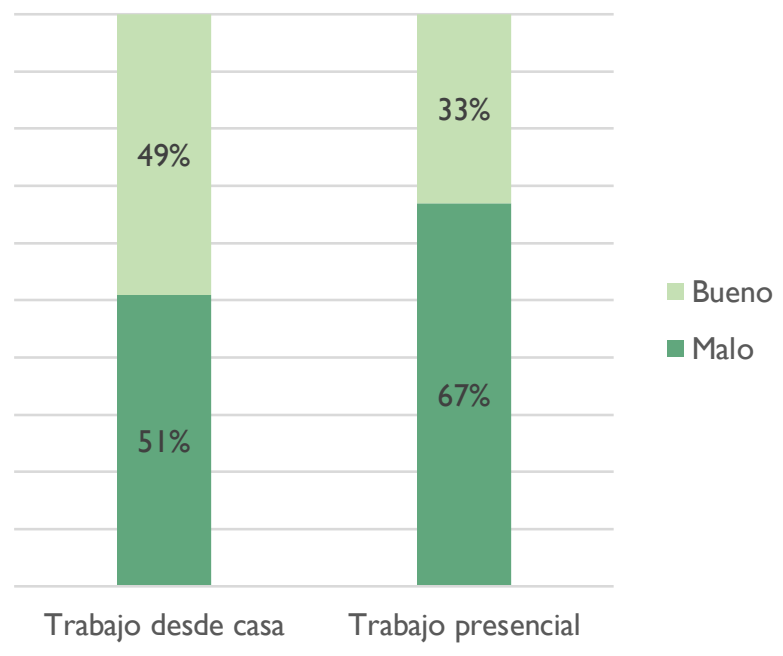

\section{Percepción de las mujeres}

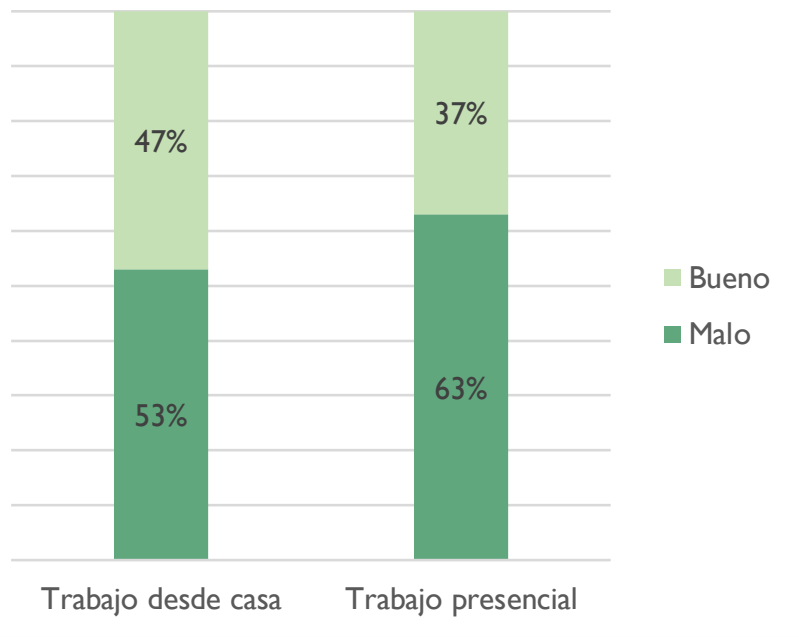

\section{Las condiciones de tiempo y productividad de las y los colaboradores en modalidad de trabajo presencial y remoto desde casa}

Entre las principales decisiones que a causa del Covid-19 están implementando las organizaciones para mantener sus operaciones productivas y comerciales están los rediseños en los puestos de trabajo y las asignaciones de nuevas tareas, en tal sentido, es importante que las empresas brinden a sus empleados 
pausas, tiempos necesarios para que puedan tomar sus alimentos y que de igual forma tengan tiempo para descansar de su jornada laboral diaria.

\section{Tabla 9}

Respeto a los tiempos de comida y horario laboral en modalidad presencial

\begin{tabular}{|c|c|c|c|c|c|c|}
\hline 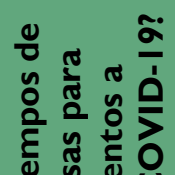 & \multicolumn{6}{|c|}{$\begin{array}{c}\text { ¿En la empresa se está respetando el } \\
\text { horario de trabajo de todos los } \\
\text { colaboradores a consecuencia del } \\
\text { COVID-19? }\end{array}$} \\
\hline 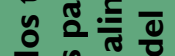 & & & \multicolumn{2}{|c|}{ Hombre } & \multicolumn{2}{|r|}{ Mujer } \\
\hline 经 & & & $\mathrm{Si}$ & No & $\mathrm{Si}$ & No \\
\hline$\stackrel{0}{0} \stackrel{0}{0}$ & \multirow{2}{*}{ Hombre } & $\mathrm{Si}$ & $42(65 \%)$ & 1 & & \\
\hline yे है & & No & 23 & 22 & & \\
\hline 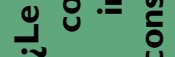 & \multirow{2}{*}{ Mujer } & $\mathrm{Si}$ & & & 23 & 6 \\
\hline & & No & & & 14 & 35 (85\%) \\
\hline
\end{tabular}

Por tal razón se les consulto a las y los colaboradores que realizan su trabajo en modalidad presencial, si se les están respetando dichas condiciones y como se puede observar en la Tabla 9, que un $65 \%$ de hombres se les respeta los horarios de trabajo y las pausas para ingerir alimentos. Por otra parte, al analizar los datos de las mujeres, al $85 \%$ no se le respeta el horario de trabajo ni los tiempos y pausas para ingerir sus alimentos.

Es muy lamentable que a la mujer no solamente se le estén incumpliendo ambas condiciones con relación al hombre, sino también que en ellas cae mayor cantidad de multitareas que realizan $y$ afectan la productividad en comparación a las que el hombre realiza. Ante lo antes expuesto es importante que los líderes y tomadores de decisiones sean flexibles y brinden a sus empleados esas pausas para que su productividad se mantenga y no los lleve a padecer enfermedades físicas, psicológicas o de salud emocional.
Otros hallazgos que se pueden resaltar sobre las condiciones de Tiempo y productividad y que se encuentras relacionadas con el personal que realiza el trabajo en modalidad remoto desde casa, son la sobrecarga de trabajo y las tareas fuera del horario laboral. En efecto las y los colaboradores manifestaron según la Tabla 10, que el $82 \%$ de los hombres perciben sobrecarga de tareas y se le solicitan también desarrollar actividades laborales fuera de su horario de trabajo, situación que es aún más alarmante para el caso de las mujeres que alcanza a un $83 \%$. En definitiva, son las mujeres las que más cargadas de actividades y solicitudes están en comparación a los hombres.

\section{Tabla 10}

Sobrecarga de tareas y solicitudes fuera del horario de trabajo en modalidad remota desde casa

\begin{tabular}{|c|c|c|c|c|c|c|}
\hline \multirow{7}{*}{ 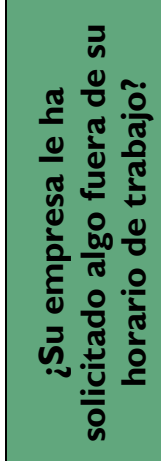 } & \multicolumn{6}{|c|}{$\begin{array}{l}\text { ¿Le están sobrecargando de tareas o } \\
\text { actividades por realizar su trabajo desde } \\
\text { casa, a consecuencia del COVID-19? }\end{array}$} \\
\hline & & & \multicolumn{2}{|c|}{ Hombre } & \multicolumn{2}{|l|}{ Mujer } \\
\hline & & & $\mathrm{Si}$ & No & $\mathrm{Si}$ & No \\
\hline & \multirow{2}{*}{ Hombre } & $\mathrm{Si}$ & 31 (82\%) & 46 & & \\
\hline & & No & 7 & 50 & & \\
\hline & \multirow{2}{*}{ Mujer } & $\mathrm{Si}$ & & & 44 (83\%) & 61 \\
\hline & & No & & & 9 & 60 \\
\hline
\end{tabular}

Siempre en la modalidad de trabajo remoto desde casa, la Figura 4 destaca que tanto a hombres como a mujeres se les está respetando las pausas para comer, los horarios laborales y los controles de trabajo; sin embargo, se puede notar que es a las mujeres (32\%) con relación a los hombres (24\%) a quienes en mayor medida no se les están respetando lo antes expuesto. 


\section{Figura 4}

Respeto de pausas, horarios laborales y supervisión

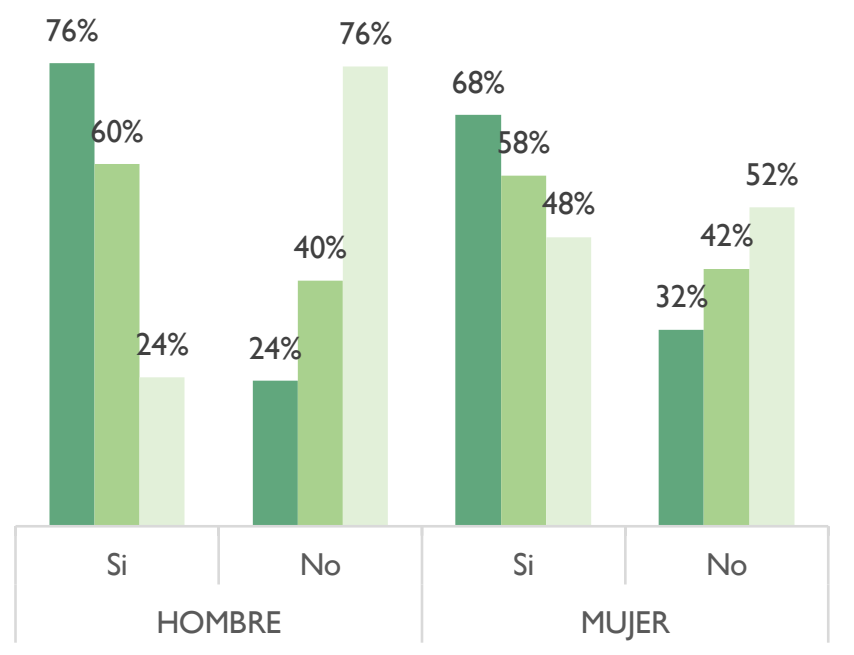

- Respetan pausas para comer

- Respetan horarios laborales

Existen controles laborales

Tomando en cuenta que ellas tienen un rol activo en casa relacionado con el cuidado de los hijos, la preparación de los alimentos, entre otras, los líderes y tomadores de decisiones de las empresas deben considerar la flexibilidad en estos escenarios, sobre todo en aquellos factores que están regulados por el Código de trabajo.

\section{Las expectativas y los temores de las y los colaboradores de las empresas salvadoreños en el contexto del Covid- 19}

En los seres humanos es normal el crearse expectativas o sentimientos de esperanza ante la posibilidad de alcanzar logros personales o grupales, así como la experimentación de la emoción, de preocupación o miedo ante aquello que represente una amenaza a la integridad física y/o psicológica de la persona.
En tal sentido, ante el eminente riesgo que representa para el desarrollo normal de la vida de cualquier persona la pandemia Covid-19, es de esperar que las y los colaboradores de las empresas salvadoreñas tengan expectativas sobre qué pasará con su situación laboral futura, así como preocupaciones sobre qué deparará la nueva normalidad tanto para sus vidas familiares como laborales.

Es en la línea de lo antes expuesto, que en los dos subapartados siguientes se presentan los resultados sobre las expectativas y las preocupaciones de las y los colaboradores de las empresas salvadoreñas en el contexto de la pandemia Covid-19, ante la reapertura de la actividad económica y la instauración de una nueva normalidad.

\section{Las expectativas de las y los colaboradores salvadoreños}

Los datos que se presentan en la Tabla II, muestran la visión que tienen las y los colaboradores sobre su situación laboral en los entornos de trabajo frente a la crisis sanitaria y económica provocada por la pandemia Covid-19.

Como se puede observar, se trata de expectativas que en su mayoría son compartidas tanto por las colaboradoras como por su contraparte masculina $y$, que tiene que ver con una visión enfocada en la conservación de la salud, que van desde acciones relacionadas con que la empresa para la cual trabajan, implemente la modalidad del Teletrabajo o cree y ejecute protocolos adecuados para continuar con las medidas sanitarias de seguridad y rediseñe los puestos de trabajo, para así evitar los riesgos de contagio.

En definitiva, sus expectativas van en la línea de que tanto en el entorno laboral, así como en el social en general, se continúe con las medidas de distanciamiento 
social; es decir, evitar estrechar la mano, saludar de beso, reuniones de grupos, entre otros; así como continuar trabajando para la empresa que les tiene contratados actualmente, debido a la situación precaria en la que se encuentra en estos momentos el mercado laboral salvadoreño.

\section{Tabla I I}

Expectativas de las y los colaboradores salvadoreños ante el Covid-19

\begin{tabular}{|c|c|c|c|c|}
\hline \multicolumn{2}{|l|}{ Expectativas } & Hombre & Mujer & \multirow{2}{*}{$\frac{\text { Total }}{25 I}$} \\
\hline $\begin{array}{l}\text { Que en la empresa para la } \\
\text { cual laboro implemente la }\end{array}$ & Si & $\begin{array}{c}114 \\
(25 \%)\end{array}$ & $\begin{array}{c}137 \\
(30 \%)\end{array}$ & \\
\hline $\begin{array}{l}\text { modalidad del trabajo desde } \\
\text { casa. }\end{array}$ & No & 92 & 118 & 210 \\
\hline \multirow{2}{*}{$\begin{array}{l}\text { Que la empresa para la cual } \\
\text { laboro tenga un protocolo } \\
\text { adecuado para continuar } \\
\text { con las medidas sanitarias } \\
\text { de seguridad. }\end{array}$} & $\mathrm{Si}$ & $\begin{array}{c}162 \\
(35 \%)\end{array}$ & $\begin{array}{c}187 \\
(41 \%)\end{array}$ & 349 \\
\hline & No & 44 & 68 & 112 \\
\hline \multirow{2}{*}{$\begin{array}{l}\text { Continuar con las medidas } \\
\text { de distanciamiento social } \\
\text { (Evitar estrechar la mano, } \\
\text { saludar de beso, reuniones } \\
\text { de grupos, entre otros). }\end{array}$} & $\mathrm{Si}$ & $\begin{array}{c}152 \\
(33 \%)\end{array}$ & $\begin{array}{c}180 \\
(39 \%)\end{array}$ & 332 \\
\hline & No & 54 & 75 & 129 \\
\hline \multirow{2}{*}{$\begin{array}{c}\text { Que la empresa para la cual } \\
\text { laboro rediseñe los puestos } \\
\text { de trabajo para evitar el } \\
\text { riesgo de contagio. }\end{array}$} & Si & $\begin{array}{c}89 \\
(19 \%)\end{array}$ & $\begin{array}{c}130 \\
(28 \%) \\
\end{array}$ & 219 \\
\hline & No & 117 & 125 & 242 \\
\hline \multirow[b]{2}{*}{ Cambiar de trabajo. } & $\mathrm{Si}$ & 10 & 19 & 29 \\
\hline & No & $\begin{array}{c}196 \\
(43 \%)\end{array}$ & $\begin{array}{c}236 \\
(51 \%) \\
\end{array}$ & 432 \\
\hline
\end{tabular}

\section{Las preocupaciones de las y los colaboradores salvadoreños}

La Tabla 12 presenta los datos que señalan las principales preocupaciones que abaten en el día a día a las y los colaboradores de las empresas en El Salvador.

Se trata de temores relacionados en su mayoría con la posibilidad de contagiarse del Covid-19 y que son compartidos, al igual que el caso de las expectativas, tanto por mujeres como por hombres. Además, manifiestan la existencia del temor de que la empresa que les tiene contratados realice recortes masivos de personal; que, sin lugar a duda, se fundamenta en la situación de crisis económica que ha provocado la pandemia Covid-19, no solamente a nivel nacional sino internacional, con lo cual se vuelve bastante probable que las organizaciones puedan enfrentar problemas en varios eslabones de su cadena de valor que los lleve a tomar la decisión de tener que despedir personal.

\section{Tabla I2}

Preocupaciones de las y los colaboradores salvadoreños ante el Covid-19

\begin{tabular}{|c|c|c|c|c|}
\hline Preocupación & & Hombre & Mujer & Total \\
\hline \multirow{2}{*}{$\begin{array}{l}\text { Estar cerca de colegas, } \\
\text { clientes, entre otros. }\end{array}$} & $\mathrm{Si}$ & $\begin{array}{c}80 \\
(17 \%)\end{array}$ & $\begin{array}{c}139 \\
(30 \%)\end{array}$ & 219 \\
\hline & No & 126 & 116 & 242 \\
\hline \multirow{2}{*}{$\begin{array}{l}\text { Recortes masivos de } \\
\text { personal. }\end{array}$} & $\mathrm{Si}$ & $\begin{array}{c}94 \\
(20 \%)\end{array}$ & $\begin{array}{c}107 \\
(23 \%) \\
\end{array}$ & 201 \\
\hline & No & 112 & 148 & 260 \\
\hline \multirow{2}{*}{$\begin{array}{c}\text { Que la empresa para la cual } \\
\text { laboro no esté preparada } \\
\text { para manejar un caso de } \\
\text { coronavirus dentro de la } \\
\text { empresa. } \\
\end{array}$} & $\mathrm{Si}$ & $\begin{array}{c}64 \\
(14 \%) \\
\end{array}$ & $\begin{array}{c}112 \\
(24 \%) \\
\end{array}$ & 176 \\
\hline & No & 142 & 143 & 285 \\
\hline \multirow{2}{*}{$\begin{array}{l}\text { Contagiarme de COVID- } \\
\qquad 19 .\end{array}$} & $\mathrm{Si}$ & $\begin{array}{c}140 \\
(30 \%)\end{array}$ & $\begin{array}{c}202 \\
(44 \%)\end{array}$ & 342 \\
\hline & No & 66 & 53 & 119 \\
\hline
\end{tabular}

\section{CONCLUSIONES}

No cabe duda de que el empoderamiento de la mujer salvadoreña y de las mujeres alrededor del mundo es necesario, para que así puedan participar plenamente de todos los sectores de la actividad económica, de modo que se propicie una sociedad más justa, equitativa y estable, así como un dinamismo económico fuerte, centrado en las personas $y$ en la sostenibilidad medioambiental, elementos que son fundamentales en la concepción de un trabajo decente para todos y todas. 
En consecuencia, tanto a nivel nacional como internacional, la actividad empresarial está sufriendo el impacto de la pandemia Covid-19, que está afectando el clima organizacional en las empresas, sin importar su tamaño, tipo o actividad productiva, en la que se observa un mayor porcentaje de mujeres que están siendo sobrecargadas tanto en las actividades laborales como en la multiplicidad de roles que desempeña en su hogar lo que les genera estrés con riesgo de padecer enfermedades físicas, emocionales y psicológicas.

De lo que se trata, es que las mujeres y los hombres gocen de las mismas condiciones $y$ oportunidades para hacer efectivos el disfrute pleno de sus derechos humanos y su potencial, a fin de contribuir al desarrollo nacional, político, económico, social y cultural y de beneficiarse de sus resultados (UNESCO, 2019).

En esa misma línea, la Constitución de la República de El Salvador (1983), en su Art. 3 señala: Todas las personas son iguales ante la ley. Para el goce de los derechos civiles no podrán establecerse restricciones que se basen en diferencias de nacionalidad, raza, sexo o religión. No se reconocen empleos ni privilegios hereditarios. Además, no hay que olvidar que el aporte de las mujeres es necesario para que las sociedades puedan iniciar procesos de desarrollo sostenible.

Con lo cual, de lo que se trata es de que como sociedad aprendamos a reconocer que tanto mujeres como hombres tienen los mismos derechos $y$ obligaciones ante la ley y romper de una vez por todas con los estereotipos de género, que representan el elemento más fuerte y rígido del sexismo y la desigualdad (Ley Especial Integral para una Vida Libre de Violencia, 2010).
En efecto, en el contexto de la crisis sanitaria y económica provocada por la pandemia Covid-1 9 son las mujeres las que lo están pasando realmente mal, dado que les está tocando ocuparse más de los niños y de los quehaceres del hogar, situación que todavía se agrava aún más si se trata de madres solteras.

También, ONU Mujeres (2020) ha advertido que las mujeres sufrirán especialmente las consecuencias económicas y de salud emocional y psicológicas de la pandemia Covid-19. Ello porque aproximadamente el $59 \%$ de las mujeres en América Latina y el Caribe trabajan en el sector informal, ganando menos que los hombres, con lo cual tienen una menor capacidad de ahorros y un mayor riesgo de caer en condición de pobreza (Mujeres ONU, 20I6).

Por ello las naciones deben propiciar modelos de Trabajo Decente que contribuyan a disminuir la pobreza e inequidad, así como la búsqueda de bienestar, poniendo al centro a la persona, especialmente en lo relacionado a su salud física, emocional y psicológica; por lo que el Trabajo Decente debería ser considerado un derecho humano.

\section{BIBLIOGRAFÍA}

Anker, R., Chernyshev, I., Egger, P., Mehran, F., y Ritter, J.A. Jun (2003). La medición del trabajo decente con indicadores estadísticos. Revista Internacional del Trabajo, 122(2), I6I-195. https://doi.org/10.1 I I I/j.1564913X.2003.tb00172.x

Asamblea Legislativa de El Salvador (I983). Constitución de la República de El Salvador.

Benedicto XVI. Vaticano II. Caritas in Veritate. Junio 20 de 2009

Cornelio Landero, Egla (2019). Derecho del migrante a un trabajo decente en México / The migrant's right to decent work in Mexico. Barataria: Revista Castellano-Manchega de Ciencias Sociales, 103. https://doi.org//0.20932/barataria.v0i25.495 
DIGESTYC (2017). Encuesta de Hogares de Propósitos Múltiple.

http://www.digestyc.gob.sv/index.php/temas/des/ ehpm/publicaciones-ehpm.html

Gálvez, E., Gutiérrez, E., Picazzo, E. y Osorio, J. (20I6). El trabajo decente, una alternativa para reducir la desigualdad en la globalización: el caso de México. Región y sociedad, 28(66), 55-94.

Guterres, A. (2020). La pandemia expone y explota desigualdades de todo tipo, incluida la de género | Naciones Unidas. United Nations. https://www.un.org/es/coronavirus/articles/guter res-covid-19-expone-desigualdad-genero

Ley especial integral para una vida libre de violencia para las mujeres. (20I0).

https://www.asamblea.gob.sv/sites/default/files/do cuments/decretos/325EF057-A460-4BCE-AFI5620CB8AD57E5.pdf6

Mujeres O.N.U. (2020). Heroínas, las mujeres en el contexto de COVID-19: Trabajo de cuidados en tiempos de pandemia. ONU Mujeres | México. https://mexico.unwomen.org/es/noticias-yeventos/articulos/2020/06/heroinas-covid 19/h2trabajo-de-cuidados-en-tiempos-de-pandemia

Mujeres, O. N. U. (20I6). El progreso de las mujeres en el mundo 2015-2016. Transformar las economías para realizar los derechos. Resumen. Revista Estudios Feministas, 24(2), 589-6I4.

Mujeres, O.N.U. (2020, 16 septiembre). La pandemia de la COVID-19 y sus efectos económicos en las mujeres: la historia detrás de los números. ONU Mujeres.

https://www.unwomen.org/es/news/stories/2020/ 9/feature-covid-19-economic-impacts-on-women

Organización Internacional del Trabajo (9 de agosto de 2004). Organización Internacional del Trabajo.

Obtenido de https://www.ilo.org/americas/salade-prensa/WCMS_LIM_653_SP/lang-es/index.htm

PNUD (I2 de marzo de 20I5). El PNUD en El Salvador.

http://www.sv.undp.org/content/el_salvador/es/h ome/ourwork/womenempowerment/in_depth.ht $\mathrm{ml}$

Pozo-Pérez, A. (20I5). Relación de productividad, motivación y la satisfacción laboral de los empleados de una empresa de seguros y su líder. Hitos de Ciencias Económico-Administrativas, $2 \mathrm{I}(60)$, I-I8. https://revistas.ujat.mx/index.php/hitos/article/vie w/1373/III5
Pravin Sinha. (2019). Bridging inequalities for decent work in South Asia. International Journal of Labour Research, 9(I/2), 235-252.

https://search.proquest.com/docview/233097066 $\underline{7}$

Reyes Sánchez, C., Del Callejo Canal, D., y Canal Martínez, M. E. (20I8). Desigualdad salarial entre hombres y mujeres en el Gobierno Federal. El caso del Servicio Exterior Mexicano. Interconectando Saberes, (6), I-I I. https://doi.org// 0.25009/is.v0i6.2590

Somavía J. (1999). 87 Reunión de la Conferencia Internacional del Trabajo. https://www.ilo.org/public/spanish/standards/relm /ilc/ilc87/rep-i.htm

Tadjoeddin, M. Z. (2019). Decent Work. On the Quality of Employment in Indonesia. Asian Journal of Social Science, 42(I-2), 9-44. https://doi.org/|0.1163/|56853|4-04201004

UNESCO (2019). Organización de las Naciones Unidas para la Educación, la Ciencia y la Cultura. Obtenido de http://www.unesco.org

Vaquerano-Amaya, J. A., Flores-Polanco, M. I., y MoránValencia, M. A. (2020, octubre). Una aproximación al clima organizacional en las empresas de El Salvador bajo el contexto de la pandemia COVID-19. Síntesis del informe. Publicaciones Universidad Centroamericana José Simeón Cañas. El Salvador, C.A. http://www.uca.edu.sv/wpcontent/uploads/2020/I I/investigacion-uca-unaaproximacion-al-clima-organizacional-covid19.pdf 